\title{
Hyperlipidemia Related to the Use of HIV-Protease Inhibitors: Natural History and Results of Treatment with Fenofibrate
}

\author{
Bruno Caramelli, Claudia Y.S.M. de Bernoche, \\ Ana M. C. Sartori, Andrei C. Sposito, \\ Raul D. Santos, Maristela C. Monachini, \\ Tania Strabelli and Davi Uip
}

Heart Institute (InCor) and AIDS Clinic, University of São Paulo Medical School, São Paulo, Brazil

\begin{abstract}
Hyperlipidemia has been frequently recorded as a side effect of treating HIV patients with protease inhibitors (PI). This study was initiated to analyze the modifications on blood lipids in HIV-patients receiving PI and the safety and efficacy of the treatment with fenofibrate. Total (TC) and HDL-cholesterol, triglycerides (TG), and $\mathrm{CD}_{4}^{+}$T-cell counts were measured in 30 HAARTnaive patients (Group I) before and after PI introduction. In a second phase of the study, the effects of fenofibrate on lipids, $\mathrm{CPK}, \mathrm{CD}_{4}^{+}$, and viral load were determined in 13 patients (Group II) with elevated TC or TG. In Group I, $60 \%$ of the patients showed TC or TG elevations. Average increments of $31 \%$ and $146 \%$ in TC and TG respectively $(p<0.0006$ and $p<0.0001)$ were observed. In Group II, fenofibrate treatment was associated with decrements of $6.6 \%$ (TC) and $45.7 \%$ (TG) (p=0.07 and 0.0002) and no modifications on $\mathrm{CPK}, \mathrm{CD}_{4}^{+}$, and viral load. In conclusion, hyperlipidemia is common during the treatment of HIV with protease inhibitors, and fenofibrate appears to be an effective and safe choice for its treatment.

Key Words: Cholesterol, lipids, HIV infection, triglycerides.
\end{abstract}

HIV-protease inhibitors (PI) are now widely used for the treatment of HIV-positive patients [1]. A reduction in morbidity as well as an increase in life expectancy was obtained in patients treated with the highly active antiretroviral therapy (HAART) [1]. However, side effects have been described, associated with the use of PI [1-9]. Hyperlipidemia and particularly severe hypertriglyceridemia have been observed in patients taking protease inhibitors although a causal relation between lipid abnormalities and PI has not been well established [2-9]. This is an important issue, considering that maintaining the

Received on 3 October 2001; revised 18 December 2001.

Address for correspondence: Dr. Bruno Caramelli, Heart Institute (InCor), Unidade de Medicina Interdisciplinar em Cardiologia. Av. Dr. Enéas C. de Aguiar 44, São Paulo, Brazil, Zip Code: 05403-900. E-mail: bcaramel@usp.br. Fax: (5511) 3069-5535. Phone:(5511)3069-5376.

The Brazilian Journal of Infectious Diseases 2001;5(6):332-338. (C) 2001 by The Brazilian Journal of Infectious Diseases and Contexto Publishing. All rights reserved.

$1413-8670$ levels of blood cholesterol and triglycerides bellow high-risk levels is an important goal for primary prevention of cardiovascular diseases [10-11].

The natural history of hyperlipidemia in HAARTnaive patients has not been established in prospective clinical trials. The incidence, prevalence, time interval to develop lipid alterations after PI introduction, and the diagnosis of the type of hyperlipidemia remain undetermined. Moreover, once the diagnosis is established, pharmacological treatment for this condition is controversial due to the risk of skeletal muscle toxicity and potential efficacy reduction related to metabolic induction leading to lower plasmatic levels of PI and virologic failure [7-8].

The greater effect on elevated levels of LDLcholesterol than older generation fibrates, associated to the reduction of plasma triglycerides makes fenofibrate a monotherapeutic option for HIVprotease inhibitors related hyperlipidemia.[12-13]. However, data on its efficacy and safety in this clinical setting are lacking. 
This study aimed to prospectively determine the modifications on blood lipids after the introduction of the therapy with HIV-protease inhibitors and to establish the efficacy and safety of the pharmacological treatment with fenofibrate in HIV-positive patients.

\section{Population and study design}

In an earlier study published elsewhere, we analyzed the lipid profile of HIV-positive patients and compared it to age and sex matched volunteers [14]. That study was initiated in 1994, before the introduction of HIVprotease inhibitors. After the introduction of these drugs, we observed patients with high levels of total cholesterol (TC) and triglycerides (TG) as well as other authors [3-6]. From this greater group, we retrospectively studied the prospective collected data regarding blood lipid levels, in order to compare data before and after PI introduction. This comprised the first phase of our study and the population is identified as Group I.

In the second phase of our study (Group II) 13 patients taking PI and showing levels of TC or TG greater than $200 \mathrm{mg} / \mathrm{dL}$ were followed during treatment with fenofibrate. This population was included in an open, non-random and non placebo controlled study of patients who received dietary treatment plus fenofibrate. Except for 3 patients, Groups I and II did not represent the same population.

Patients showing low compliance of medication, those who used or had previous use of any drug that decreases blood lipid levels, those with liver or renal disease, or diabetes mellitus were excluded after diagnosis by history, treatment, or laboratory analysis.

The study protocol was approved by the ethical committee on human experimentation from the Hospital das Clínicas da Universidade de São Paulo.

\section{Methods}

Blood lipids: Fasting blood samples were obtained from patients before (baseline sample) and at least one month after the start of HIV-protease inhibitor (PI sample) in Group I and before and after the introduction of fenofibrate treatment in Group II. The concentrations of TC, TG and HDL-cholesterol were obtained through enzymatic colorimetric methods.

Patients were classified as having one of the predefined types of hyperlipidemia: isolated hypercholesterolemia (TC $>200 \mathrm{mg} / \mathrm{dL}$ and TG $<200$ $\mathrm{mg} / \mathrm{dL}$ ), isolated hypertriglyceridemia (TC $>200 \mathrm{mg} /$ $\mathrm{dL}$ and $\mathrm{TG}<200 \mathrm{mg} / \mathrm{dL}$ ) or combined hyperlipidemia ( $\mathrm{TC}$ and $\mathrm{TG}>200 \mathrm{mg} / \mathrm{dL}$ ).

Treatment for hyperlipidemia: For the purpose of this study, levels of TC and/or TG $>200 \mathrm{mg} / \mathrm{dL}$ associated to the use of HIV-protease inhibitors were considered elevated and patients (Group II) received dietary recommendations according to the National Cholesterol Education Program (NCEP) guidelines plus micronized fenofibrate $200 \mathrm{mg}$ daily [11].

Efficacy and safety of pharmacological treatment: Efficacy of the treatment was determined by the capacity of diet plus fenofibrate to reduce lipids to desired levels (TC $<200 \mathrm{mg}$ / $\mathrm{dL}$ and $\mathrm{TG}<200 \mathrm{mg} / \mathrm{dL}$ ). Safety was evaluated by the determination of creatine kinase (CK) levels (enzymatic ultraviolet method), $\mathrm{CD}_{4}^{+} \mathrm{T}$-cell counts and plasma HIV-1 RNA load (viral load) during drug treatment for PI related hyperlipidemia. Liver enzymes levels were also obtained for all patients included in Group II, after treatment with fenofibrate.

Plasma HIV-1 RNA determination: Viral load was measured in all Group II patients by NASBA (Organon Technika). The number of HIV-1 RNA copies was calculated on the basis of the manufacturer's reference standards.

PI regimen: The HIV-protease inhibitor regimen considered was the one taken at least one month before the time of PI sample analysis for Group I and, for Group II, the drugs taken at the time of fenofibrate introduction. 


\section{Statistical analysis}

Baseline values were compared to data obtained after treatment for Groups I and II. For weight, total and HDL-cholesterol comparisons, two-tailed student t-test was adopted. For triglycerides and $\mathrm{CD}_{4}^{+} \mathrm{T}$-cell counts, two-tailed Wilcoxon signed-rank test was used. Logarithmic transformation was adopted for plasma HIV-1 RNA load values and summarized by use of means and standard deviations. Statistical analysis was then performed by two-tailed Wilcoxon signed-rank test.

When appropriate, the frequencies of each type of hyperlipidemia before and after treatment were compared by Fisher's exact test. The level of significance accepted for all tests was 0.05 .

\section{Results}

\section{Group I}

Clinical Characteristics. Thirty patients (24 men and 6 women) were included in Group I. The mean age was 39.6 years old and ranged from 23 to 67 years old. Twenty-one patients (70\%) were taking indinavir, $6(20 \%)$ were on HCG-saquinavir, $2(6.6 \%)$ on ritonavir, and 1 (3.3\%) on ritonavir plus HCGsaquinavir as the HIV-protease inhibitor regimen considered. The average time interval between baseline and the sample obtained after PI introduction was 203 days.

No significant modifications were observed on weight after the introduction of PI $(72.5 \pm 16.9$ to $72.3 \pm 16.0$ $\mathrm{kg})$. Regarding $\mathrm{CD}_{4}^{+} \mathrm{T}$-cell counts, however, there was an increment related to the introduction of PI (159 \pm 155 to $345 \pm 247$ cells $/ \mu \mathrm{L}, \mathrm{p}<0.0001$ ) (Table 1 ).

Blood lipids. Treatment with PI was associated with average increments of $31 \%$ for TC and $146 \%$ for TG. No significant modifications were observed in HDLcholesterol levels (Table 1).

At inclusion, there were $23 \%$ of the patients had increased levels of TC, $26 \%$ with increased TG, and $36 \%$ needed treatment for any lipid alteration. After the introduction of PI, $43 \%$ of the patients showed elevated TC, $53 \%$ elevated TG $(p=0.17$ and $p=0.06$ respectively), and $60 \%$ had to be treated for any lipid alteration $(\mathrm{p}=0.12)$.

Regarding the type of hyperlipidemia, before treatment, isolated hypercholesterolemia, hypertriglyceridemia and combined hyperlipidemia corresponded to $10 \%, 13 \%$, and $13 \%$ of the patients respectively. After treatment, prevalences were $6 \%$, $16 \%$, and $36 \%$, respectively ( $p=0.07$ for combined hyperlipidemia).

\section{$\underline{\text { Group II }}$}

Clinical characteristics. Thirteen patients (11 men and 2 women) were included in Group II. The mean age was 43 (31-66) years old. Five patients (38.4\%) were taking ritonavir plus HCG-saquinavir, $4(30.7 \%)$ were on indinavir, $2(15.3 \%)$ on ritonavir, and $2(15.3 \%)$ on nelfinavir as the HIV-protease inhibitor regimen considered.

No significant modifications were observed on weight, $\mathrm{CD}_{4}^{+} \mathrm{T}$-cell counts, and viral load in Group II, after the introduction of fenofibrate (Table 2). For all patients, $\mathrm{CK}$ and liver enzymes levels remained within the normal range.

Blood lipids. After an average time of 36 days (11 to 103 ) of treatment with fenofibrate, we observed an average decrement of $6.66 \%$ for TC and $45.70 \%$ for TG. For HDL-cholesterol, an average increment of $21.30 \%$ was observed (Table 3 ).

There was a non-significant decrease on the prevalence of elevated levels of TC ( $84 \%$ to $69 \%$, $\mathrm{p}=0.64)$ and a significant decrement on the prevalence of elevated levels of TG $(100 \%$ to $61 \%$, $\mathrm{p}=0.03)$. After treatment, $76 \%$ of the patients still did not show desired levels of TC and TG. The prevalences of the types of hyperlipidemia before fenofibrate treatment were $0 \%, 15 \%$, and $84 \%$ for hypercholesterolemia, hypertriglyceridemia, and combined hyperlipidemia respectively. After treatment, prevalences were, respectively, $15 \%, 7 \%$, and $53 \%$ (non significant changes). 
Table 1. Group I. Weight, $\mathrm{CD}_{4}^{+} \mathrm{T}$-cell counts, total cholesterol, triglycerides and HDL-cholesterol before and after treatment, and average increment related to the use of protease inhibitors

\begin{tabular}{lcccc}
\hline Variable & Baseline & PI & $\begin{array}{c}\text { Average } \\
\text { increment }\end{array}$ & $\mathbf{P}^{*}$ \\
\hline Weight $(\mathrm{kg})$ & $72.50 \pm 16.92$ & $72.37 \pm 16.07$ & - & 0.92 \\
$\mathrm{CD}_{4}(\mathrm{cells} / \mu \mathrm{L})$ & $159 \pm 155$ & $345 \pm 247$ & $116 \%$ & $<0.0001$ \\
Total-Cholesterol & $164 \pm 43$ & $204 \pm 63$ & $31 \%$ & $<0.0006$ \\
Triglycerides & $131(61-650)$ & $216(80-1730)$ & $146 \%$ & $<0.0001$ \\
HDL-Cholesterol & $33 \pm 9$ & $33 \pm 8$ & - & 0.95 \\
\hline
\end{tabular}

$\mathrm{CD}_{4}=\mathrm{CD}_{4}^{+} \mathrm{T}$-cell count. Data are expressed in mean values \pm standard deviation (median and range for triglycerides). Baseline $=$ values before treatment; PI $=$ values after treatment with protease inhibitor. Total and HDL-cholesterol, triglycerides are expressed in $\mathrm{mg} / \mathrm{dL}$.

* Two tailed student t-test was adopted for total and HDL-cholesterol comparisons. For triglycerides, two-tailed Wilcoxon signed-rank test was used.

Table 2. Group II. Weight, $\mathrm{CD}_{4}^{+} \mathrm{T}$-cell counts, and plasma viral load before and after fenofibrate treatment for hyperlipidemia related to the use of protease inhibitors

\begin{tabular}{lccc}
\hline Variable & Before & After & $\mathbf{P}^{*}$ \\
\hline Weight $(\mathrm{kg})$ & $69.09 \pm 9.95$ & $68.10 \pm 10.01$ & 0.16 \\
$\mathrm{CD}_{4}(\mathrm{cells} / \mu \mathrm{L})$ & $389 \pm 156$ & $412 \pm 186$ & 0.49 \\
Viral load & $2.64 \pm 1.01$ & $2.84 \pm 1.25$ & 0.49
\end{tabular}

Data are expressed in mean values \pm standard deviation (after logarithmic transformation for viral load). $\mathrm{CD}_{4}=\mathrm{CD}_{4}^{+} \mathrm{T}$-cell count.

${ }^{*}$ Two tailed student t-test was adopted for weight comparison. For $\mathrm{CD}_{4}^{+} \mathrm{T}$-cell counts and plasma HIV-1 RNA load values (after logarithmic transformation), two-tailed Wilcoxon signed-rank test was used.

Table 3. Group II. Total cholesterol, triglycerides and HDL-cholesterol, and average modification before and after fenofibrate treatment for dyslipidemia related to the use of protease inhibitors

\begin{tabular}{lcccc}
\hline Variable & Before & After & $\begin{array}{c}\text { Average } \\
\text { modification }\end{array}$ & $\mathbf{P}^{*}$ \\
\hline Total-Cholesterol & $246 \pm 61$ & $226 \pm 53$ & $-6.66 \%$ & 0.07 \\
Triglycerides & $486(314-720)$ & $274(64-478)$ & $-45.7 \%$ & 0.0002 \\
HDL-Cholesterol & $32 \pm 10$ & $38 \pm 12$ & $+21.3 \%$ & $<0.0001$ \\
\hline
\end{tabular}

Data are expressed in mean values \pm standard deviation (median and range for triglycerides) and in $\mathrm{mg} / \mathrm{dL}$.

${ }^{*}$ Two tailed student t-test was adopted for total and HDL-cholesterol comparisons. For triglycerides, two-tailed Wilcoxon signed-rank test was used. 
Table 4. Group II. Age, total cholesterol, triglycerides, HDL-cholesterol, viral load, $\mathrm{CD}_{4}{ }^{+} \mathrm{T}$-cell count, and the duration of treatment before and after treatment with fenofibrate

\begin{tabular}{ccccccccccccc}
\hline Patient & Age & TC 1 & TG1 & HDL1 & Load 1 & CD 1 & Time & TC 2 & TG 2 & HDL 2 & Load 2 & CD 2 \\
\hline 1 & 66 & 320 & 720 & 39 & 2.66 & 183 & 11 & 323 & 444 & 42 & 2.46 & 328 \\
2 & 38 & 135 & 516 & 26 & 1.90 & 447 & 28 & 146 & 175 & 30 & 5.30 & 308 \\
3 & 45 & 350 & 454 & 56 & 1.90 & 657 & 41 & 294 & 193 & 68 & 1.90 & 812 \\
4 & 39 & 236 & 599 & 20 & 4.43 & 357 & 25 & 301 & 478 & 36 & 1.90 & 312 \\
5 & 48 & 177 & 333 & 30 & 1.90 & 546 & 32 & 156 & 133 & 35 & 1.90 & 738 \\
6 & 35 & 203 & 328 & 26 & 3.27 & 218 & 103 & 191 & 157 & 30 & 3.49 & 171 \\
7 & 33 & 204 & 598 & 26 & 1.90 & 248 & 28 & 185 & 331 & 34 & 1.90 & 503 \\
8 & 56 & 293 & 484 & 31 & 1.90 & 633 & 20 & 235 & 346 & 29 & 3.14 & 319 \\
9 & 45 & 226 & 424 & 37 & 1.90 & 343 & 28 & 234 & 245 & 39 & 2.11 & 410 \\
10 & 45 & 226 & 386 & 24 & 1.90 & 228 & 35 & 205 & 255 & 42 & 1.90 & 308 \\
11 & 31 & 241 & 314 & 50 & 4.11 & 332 & 27 & 210 & 64 & 59 & 4.00 & 262 \\
12 & 38 & 306 & 609 & 34 & 4.30 & 484 & 38 & 238 & 340 & 36 & 5.11 & 520 \\
13 & 42 & 287 & 564 & 26 & 2.30 & 392 & 64 & 231 & 409 & 24 & 1.90 & 366 \\
\hline
\end{tabular}

$\mathrm{TC}=$ total cholesterol; $\mathrm{TG}=$ triglycerides; $\mathrm{HDL}=\mathrm{HDL}$-cholesterol; $\mathrm{Load}=$ Viral load; $\mathrm{CD}_{4}=\mathrm{CD}_{4}^{+} \mathrm{T}$-cell counts; $1=$ before treatment data; $2=$ after treatment data; Time=time interval between 1 and 2. Data are expressed in years (Age); mg/dL (CT; TG; HDL); copies $/ \mathrm{mL}$ after logarithmic transformation (Viral load); cells $/ \mu \mathrm{L}\left(\mathrm{CD}_{4}^{+} \mathrm{T}\right.$-cell counts); days (Time).

\section{Discussion}

Reduced levels of TC, HDL-cholesterol and elevation of TG were observed in earlier studies analyzing HIV-infected patients before the introduction of HIV-protease inhibitors [14-17]. In a cohort of 120 HIV-infected patients compared to seronegative controls, we found lower TC $(171 \pm 3 \mathrm{mg} / \mathrm{dL}$ and 185 $\pm 6 \mathrm{mg} / \mathrm{dL})$, higher TG $(159 \pm 7$ and $114 \pm 9 \mathrm{mg} / \mathrm{dL})$ and lower HDL-cholesterol (34 \pm 1 and $50 \pm 2 \mathrm{mg} /$ dL) [14].

Several non-exclusive mechanisms have been postulated to explain these findings including alterations in lipoprotein metabolism produced by cytokines involved on the immune response to infection. Hypertriglyceridemia could be a result of an increase in VLDL-cholesterol synthesis or a reduction on the hydrolysis of chylomicrons and VLDL by lipoprotein lipase. Heavy alcohol consumption, nutritional status, and diet modifications also figure as possible causes for these findings [14].
In the present study, we found a similar lipid profile in HAART-naive patients. The introduction of HIVprotease inhibitors was followed by an elevation is $\mathrm{CD}_{4}^{+}$ T-cell counts, an average $31 \%$ elevation of totalcholesterol, and a marked $146 \%$ average increment on triglycerides in the absence of significant modifications on weight that could explain it. As previously observed, these findings are part of a highly prevalent syndrome that includes a peripheral fat wasting called lipodystrophy, central adiposity, breast hypertrophy in women and insulin resistance or type 2 diabetes [9].

The pathogenesis of this syndrome still remains unknown but some authors suggest that it could be related to the homology of the catalytic region of HIV-1 protease, the site to which PI bind, to regions within two proteins that are involved in lipid metabolism: cytoplasmic retinoic-acid binding protein type 1 (CRABP-1) and low density lipoprotein-receptor-related protein (LRP). According to this hypothesis, protease inhibitors will 
inhibit CRABP-1 and bind to LRP leading to the metabolic and clinical findings in the syndrome, including hyperlipidemia [5].

Hyperlipidemia, which has been observed as early as a few weeks after the introduction of HIV-protease inhibitors, became a major concern because of its association with atherosclerosis [9]. In fact, several recent case-reports described cardiovascular events in young patients taking PI and showing high levels of TC and TG in the absence of other risk factors [2,4-5].

The finding that $60 \%$ of patients with elevated levels of TC and/or TG in our study and that combined hyperlipidemia is the most common type of alteration observed, addresses the question if nutritional and/or pharmacological treatment for this specific condition is effective and safe.

The treatment for HIV-protease inhibitors related hyperlipidemia has gained great interest in the literature and therapeutic approaches were proposed. Dietary modifications could be difficult to achieve in this population with frequent gastrointestinal symptoms and fear of weight loss. Great concern has been expressed regarding the pharmacological treatment in this situation: there is a potential risk of skeletal muscle toxicity due to increased plasma levels of statins caused by CYP3A4 inhibition by PI, and of lower serum levels of PI related to p450 induction by these drugs, leading to virologic failure [7-8].

A fibrate plus a statin could be the best option for treatment, as combined hyperlipidemia often requires the association of these drugs. However, only small and no prospective studies were reported [6]. Limited data suggest that atorvastatin and gemfibrozil are effective in lowering both cholesterol and triglycerides although these lipids remained frequently above recommended levels in spite of treatment [6].

Fenofibrate might be an alternative to the statinfibrate association in the treatment of HIV-protease inhibitor induced hyperlipidemia. Actually, by reducing both triglycerides and LDL-cholesterol without statin administration, fenofibrate might have safety and cost advantages in this situation. A previous report described the use of fenofibrate in two HIV-positive patients, provoking a marked decrease on triglycerides but still above recommended levels [17].
Although in a small and non-randomized pilot study, we observed an increase in HDL-cholesterol levels and a decrease in TG and TC, after the introduction of fenofibrate. In spite of the still high prevalence of patients not showing desired levels of blood lipids (76\%), treatment with fenofibrate was associated with a "less atherogenic" lipid profile. Moreover, the fact that no patient showed elevation of $\mathrm{CK}$ and no significant variations were observed on $\mathrm{CD}_{4}^{+} \mathrm{T}$-cell counts and viral load suggests that this treatment is safe.

Finally, considering the 7 patients with moderate hypertriglyceridemia (TG between $200-500 \mathrm{mg} / \mathrm{dL}$ ), all but one showed normal or near normal levels of triglycerides after treatment (Table 4).

\section{Clinical implications}

The increment in life expectancy after the introduction of HIV-protease inhibitors will certainly increase the period of time for vascular endothelium to be exposed to a high lipidic, atherogenic milieu associated with the use of these drugs. This phenomenon could lead to an elevation in the incidence of cardiovascular events like an acute myocardial infarction or unstable angina. The results of primary and secondary atherosclerosis prevention studies, demonstrating the benefit of statins or fibrates, allied to the increase in life expectancy observed after the use of PI in HIV-patients might be a reason for the treatment of hyperlipidemia in this population [7,8,10-11].

The simple change from one protease inhibitor to another does not seems to be the right choice, as conclusive data about lipid levels modifications associated with different PI regimens are lacking. Conversely, future drugs should be studied looking for different or no effects on lipoprotein metabolism.

Diet and, if necessary, pharmacological treatment has been recommended $[7,8]$. However, once treatment is started, we still need to know for how long and if recommended levels are the same for this younger and, theoretically, lower risk population. All of these issues must be addressed on future clinical trials involving a greater number of patients. 
Fenofibrate seems to be an effective and safe choice for patients with moderate hyperlipidemia related to the use of HIV-protease inhibitors.

\section{References}

1. Carpenter C.C.J., Fischl M.A., Hammer S.M., et al. Antiretroviral therapy for HIV infection in 1998. JAMA 1998;280:78-86.

2. SoRelle R. Vascular and lipid syndromes in selected HIVinfected patients. Circulation 1998;98:829-30.

3. Lo J.C., Mulligan K., Tai V.W., et al. "Buffalo hump" in men with HIV-1 infection. Lancet 1998;351:867-70.

4. Henry K., Melroe H., Huebsch J., et al. Severe premature coronary artery disease with protease inhibitors. Lancet 1998;351:1328.

5. Carr A., Samaras K., Chisholm D.J., Cooper D.A. Pathogenesis of HIV-protease inhibitor-associated peripheral lipodystrophy, hyperlipidemia, and insulin resistance. Lancet 1998;351:1881-3.

6. Henry K., Melroe H., Huebesch J., et al. Atorvastatin and gemfibrozil for protease-inhibitor-related lipid abnormalities. Lancet 1998;352:1031-1032.

7. Penzak SR, Chuck SK. Hyperlipidemia associated with HIV protease inhibitor use: pathophysiology, prevalence, risk factors and treatment. Scand J Infect Dis 2000;32:111-23.

8. Dubé M.P., Sprencher D., Henry W.K., et al. Preliminary guidelines for the evaluation and management of dyslipidemia in HIV-Infected adults receiving antiretroviral therapy. Recommendations of the Adult ACTG Cardiovascular disease Focus group. Clin Infect Dis 2000;31(5):1216-24.

9. Carr A., Samaras K., Thorisdottir A., et al. Diagnosis, prediction, and natural course of HIV-1 protease-inhibitorassociated lipodystrophy, hyperlidemia, and diabetes mellitus: a cohort study. Lancet 1999;353:2093-9.

10. Shepherd J., Cobbe S.M., Ford I., et al. Prevention of coronary heart disease with pravastatin in men with hypercholesterolemia. West of Scotland Coronary Prevention Study Group. N Eng J Med 1995;333:1301-7.

11. Summary of the Second Report of the National Cholesterol Education Program (NCEP). Expert Panel on Detection, Evaluation and Treatment of high Cholesterol in Adults (Adult treatment Panel II). JAMA 1993;269:3015-23.

12. Miller D.B., Spence J.D. Clinical Pharmacokinetics of fibric acid derivatives (fibrates). Clin Pharmacokinetic 1998;34:155-62.

13. Packard C.J. Overview of fenofibrate. Eur Heart J 1998; 19 Suppl A:A62-5.
14. Sposito A.C., Caramelli B., Sartori A.M., Ramires J.A.F. The lipoprotein in HIV infected patients. The Brazilian Journal of Infectious Diseases 1997; 1:275-83.

15. Grunfeld C., Pang M., Doerrler W., et al. Lipids, lipoproteins, triglycerides clearance, and cytokines in human immunodeficiency syndrome. J Clin Endocrinol Metab 1992;74:1045-52.

16. Constans J., Pellegrin J.L., Peuchant E., et al. Plasma lipids in HIV-infected patients: a prospective study in 95 patients. European Journal of Clinical Investigation 1994;24:416-20.

17. Thomas J.C., Lopes-Virella M.F., Del Bene V.E., et al. Use of fenofibrate in the management of protease inhibitorassociated lipid abnormalities. Pharmacotherapy 2000;20(6):727-34. 